\title{
Correlation analysis of compromised immune function with perioperative sepsis in HIV-positive patient
}

\author{
Baochi Liu*, Meng Wang, Jinsong Su, Yanzheng Song, Li Liu, Lei Li \\ Department of Surgery, Shanghai Public Health Clinical Center Affiliated to Fudan University, Shanghai, China; \\ *Corresponding Author: liubaochi2002@yahoo.com.cn, \{wangmengnh, sujinsong030124, yanzhengsong\}@163.com, \\ lilimx@sina.com, lilei@shaphc.org
}

Received 10 May 2011; revised 1 July 2011; accepted 15 July 2011

\begin{abstract}
Objective: To investigate the relationship between immune function and perioperative sepsis in HIVpositive patients. Methods: Retrospective analysis of $144 \mathrm{HIV}$-positive patients surgically treated from Oct 2008 to Dec 2010 in Shanghai Public Health Clinical Center. The patients were divided into four groups based on their $\mathrm{CD4}^{+} \mathrm{T}$ cells counts in preoperative period: group A (0 - 99 cell/ul), group B (100 - 199 cell/ul), group C (200 349 cell/ul), group $D(\geq 350 \mathrm{cell} / \mathrm{ul})$. All patients had received standardized surgical procedures, careful surgical routines were applied. To reduce operational damage, conventional antibiotics, anti-TB, anti-fugal, antiretroviral therapies were used to prevent infection and promptly treatment of complications. Results among 144 HIV-positive patients (male 133 and female 11, aged $42.6 \pm 12.5$ ), 80 patients got perioperative sepsis (14 cases in preoperative period and 66 cases in postoperative period). 64 cases did not get sepsis. The average $C D 4^{+} T$ cell count was $276.97 \pm 137.91$ in preoperative period of patients without sepsis, which was significant higher than the patients with preoperative sepsis $(151.29 \pm 110.64)$ and patients with postoperative sepsis(161.14 \pm 128.45$)(F=13.91, P<0.05)$. There were statistic differences of perioperative sepsis among A group (82.6\%), B group (69.8\%), $C$ group (40.5\%) and D group (26.9\%) $\left(X^{2}=23.680\right.$, $P<0.01)$. Three cases died of MODS in group A, each one died of MODS in group $B$ and group $C$. There was no death in group $D$. There was negative correlation between the incidence of sepsis and levels of $C D 4^{+} T$ cells $(r=-0.987, P=$ 0.013). Conclusions With the decrease of preoperative $\mathrm{CD}^{+} \mathrm{T}$ cells, the risk of perioperative
\end{abstract}

sepsis had notable increase. Complete evaluation of surgical risk and suitable perioperative treatments may obtain better effect for the patients infected HIV.

Keywords: HIV; Acquired Immunodeficiency Syndrome; Sepsis; Surgical Operation

\section{INTRODUCTION}

Sepsis is a common complication following serious trauma/burn, shock, infection or major surgery, and also is a systemic inflammation response syndrome (SIRS) in the presence of infectious factors. Furthermore, sepsis can develop into septic shock and multiple organ dysfunction syndrome (MODS), which is largely responsible for the extremely high mortality. Nowadays, although great progress has been made in the pathogenesis, diagnosis and prevention of sepsis, the incidence and mortality rate of sepsis still remain high. Studies reveal the mortality rate of sepsis is as high as $28 \% \sim 56 \%$ [1-4]. Once infected with human immunodeficiency virus (HIV), humans will develop immune dysfunction. HIV infected patients with surgical conditions require surgical treatment in certain environments. Because of the immune dysfunction, HIV infected patients are susceptible to postoperative sepsis that go along with different clinical manifestations. At present plenty of comprehensive hospitals take the HIV testing as a regular program before surgery. Once HIV infection test index is positive, patients will be euphemistically advised against/denied operations by surgeons. One of the reasons against the surgical operation is that patients with HIV infection are susceptible to infectious complication after surgery.

Shanghai Public Health Clinical Center is a designated hospital for treatment of HIV infectious patients. From October 2008 to December 2010, a total of 156 HIV infected patients underwent operation at surgery depart- 
ment, among whom 144 complete cases data before and after surgery were collected. The data revealed a total of 80 cases suffered from perioperative sepsis complication. Herein, we report our remedy experiences in these patients.

\section{PATIENTS AND METHODS}

\subsection{Clinical Data}

A total of $156 \mathrm{HIV}$ infected patients who were recruited from October 2008 to December 2010 underwent surgery in the Surgery Department of Shanghai Public Health Clinical Center affiliated to Fudan University. Among those cases, 144 complete clinical data before and after surgery were subjected to retrospective analysis. The inclusion criteria included: 1) patients with HIV infection which was confirmed by the Centers of Disease Control. 2) patients with complete medical record: general examinations and examination of immune function before and after surgery which were carried out in the department of surgery of Shanghai Public Health Clinical Center. The age of these patients ranged from 19 to 74 years with an average age of $42.62 \pm 12.51$ years. There were 133 males $(92.3 \%)$ with an average age of $42.76 \pm$ 12.79 years and 11 females $(7.7 \%)$ with an average age of $40.91 \pm 8.65$ years. 80 cases suffered from perioperative sepsis complication, namely the SIRS with infective factors according to sepsis diagnosis standard from the 2001 international sepsis meeting.

\subsection{Type of Surgery and Perioperative Management}

Routine inspection was conducted after admission, On top of which operative risk was evaluated and surgical preparation was formulated prior to surgery. There were 46 gastrointestinal surgeries, 11 hepatobiliary surgeries, 18 orthopedic surgeries, 9 urological surgeries, 32 surgeries for surface mass resection, 10 surgeries for abscess incision and drainage, 7 surgeries for splenectomy, 6 surgeries for thyroid tumor resection, and 5 surgeries for other conditions such as vascular surgery and ophthalmology. There were 14 cases with preoperative sepsis. For patients with general pathogenic bacteria or with tuberculosis or cryptococcus infection before surgery, symptomatic treatment was carried out and infection was controlled. While, for patients with peritonitis or deep abscess, emergency surgery will be performed on the basis of anti-infection treatment. The vital signs were monitored and complications were timely treated after surgery. Meanwhile, nutrition support, anti-infection, anti-retroviral and anti-tuberculosis treatments also continued.

\subsection{Grouping}

Assuming there are different susceptibility and severity to the sepsis, patients were divided into 4 groups in accordance with the level of $\mathrm{CD}^{+} \mathrm{T}$ lymphocytes. Group A: CD4 T cells $<100$ cells/ $\mu \mathrm{l}$; Group B: 100 cells $/ \mu \mathrm{l} \leq$ CD4 T cells $<200$ cells/ $\mu$; Group C: 200 cells/ $\mu 1 \leq$ CD4 $\mathrm{T}$ cells $<350$ cells $/ \mu \mathrm{l}$; Group D: CD4 T cells $\geq 350$ cells $/ \mu 1$. Patients were divided into 3 groups according to preoperative or postoperative sepsis complication, no sepsis, before and after operation. The number of $\mathrm{CD}^{+} \mathrm{T}$ and $\mathrm{CD}^{+} \mathrm{T}$ cells, the ratio of $\mathrm{CD} 4^{+} \mathrm{T} / \mathrm{CD} 8^{+} \mathrm{T}$ cells and the alteration of routine blood testing were respectively statistically analyzed to explore pathogenesis regularity of perioperative sepsis in the patients with varying degrees of immune function damage.

\subsection{Statistical Analysis}

All analyses were performed with SPSS 17.0 statistical software. Sepsis is a systemic inflammation response syndrome (SIRS) in the presence of infectious factors. HIV infected patients meeting the standard of SIRS were diagnosed as sepsis, while patients with dysfunctional organs no less than two were classified as multiple organ dysfunction syndrome (MODS). Means \pm standard deviation were adopted for quantitative data, chi square test were employed for variance analysis in qualitative data. Pearson correlation was tested for linear correlation. A value less than 0.05 were considered statistically significant.

\section{RESULTS}

There were 14 cases with preoperative sepsis, 66 cases with postoperative sepsis and 64 cases without sepsis after surgery among 144 HIV infected patients. Surgery types and patients with or without preoperative sepsis are listed (Figure 1). Patients were divided into 3 groups according to preoperative or postoperative sepsis complication, no sepsis complication. The average count of $\mathrm{CD} 4{ }^{+} \mathrm{T}$ lymphocytes in the group of no perioperative sepsis is significantly higher than that of $\mathrm{CD}^{+} \mathrm{T}$ lymphocytes in the groups of preoperative and postoperative sepsis $(P<0.05)$. The levels of $\mathrm{CD}^{+} \mathrm{T}$ cells before surgery in each group are listed (Figure 2). Patients were divided into 4 groups according to the number of $\mathrm{CD}^{+} \mathrm{T}$ lymphocytes before surgery. There were 19 cases with complication of sepsis among 23 patients in the Group A $(82.609 \%), 37$ cases with complication of sepsis among 53 patients in the Group B $(69.811 \%), 17$ cases with complication of sepsis among 42 patients in the Group C $(40.476 \%)$ and 7 cases with complication of sepsis among 26 patients in the Group D $(26.923 \%)$. $\mathrm{CD}^{+} \mathrm{T}$ lymphocytes level is significantly reversely correlated 


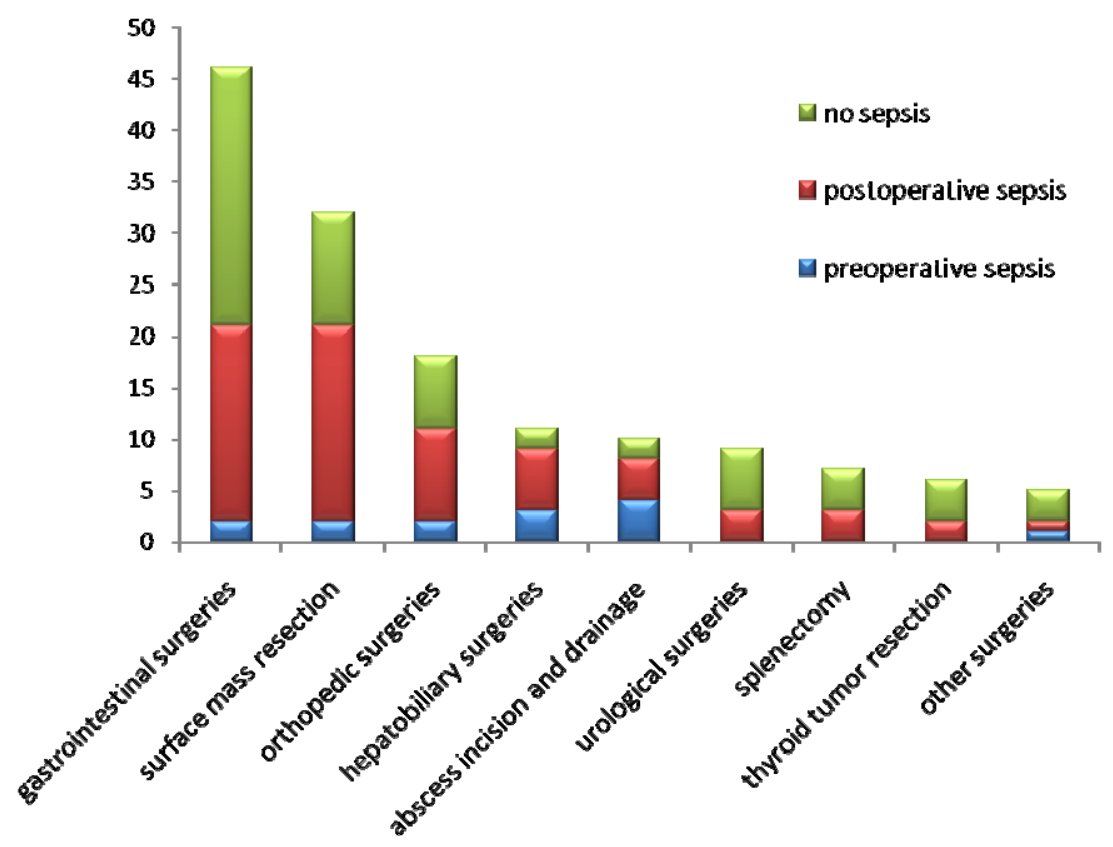

Figure 1. Surgery types and septic cases during perioperative period.

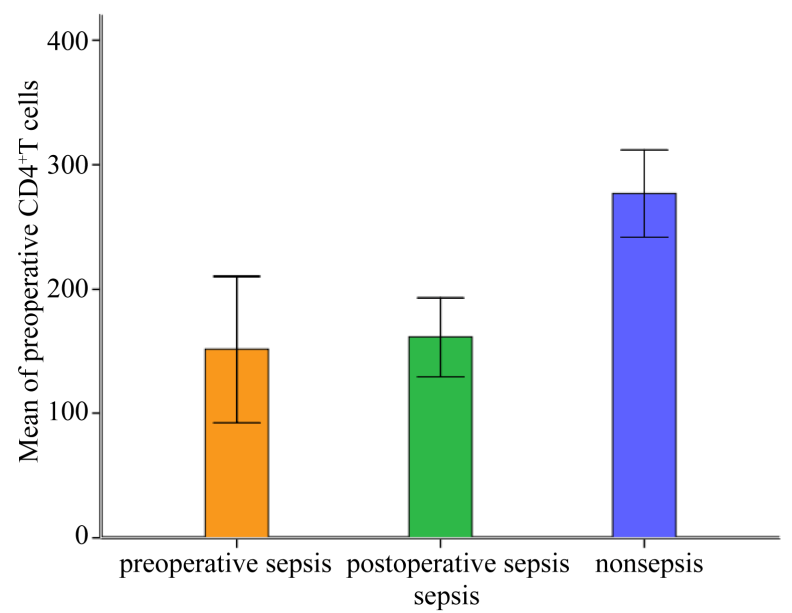

Figure 2. $\mathrm{CD}^{+} \mathrm{T}$ lymphocytes count before surgery and sepsis in each group.

with sepsis $(P<0.05)$. The linear correlation between the level of $\mathrm{CD}^{+} \mathrm{T}$ lymphocytes and the sepsis are showed (Figure 3). The relationship between the level of $\mathrm{CD}^{+} \mathrm{T}$ lymphocytes before surgery and perioperative sepsis occurrence is analyzed by adopting columns chi-square and Pearson correlation analysis (Table 1), the single factor of variance analysis of sepsis are presented in Table 2 .

\section{DISCUSSION}

\subsection{The Septic Feature of HIV-Infective Patients}

Sepsis is a systemic inflammation response syndrome

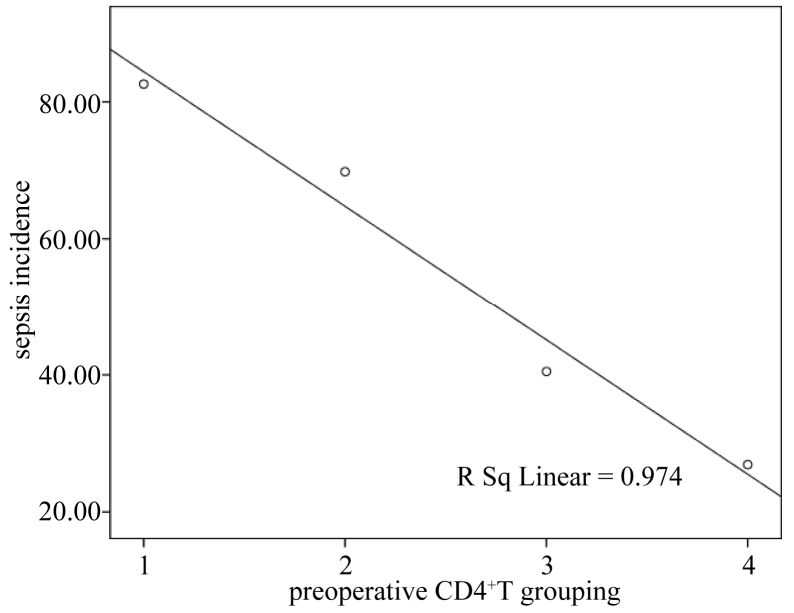

Figure 3. The linear correlation between the level of $\mathrm{CD}^{+} \mathrm{T}$ lymphocytes and sepsis.

(SIRS) in the presence of infectious factors according to sepsis diagnosis standard from the 2001 international sepsis meeting [5]. HIV infective patients were diagnosed as sepsis once meeting the standard of SIRS. The clinical manifestations of patients with HIV infection are variable. Some patients had sepsis before surgery, while some patients showed typical clinical manifestations of SIRS after surgery, such as fever, accelerated heartbeat, fast breath and increased number of White blood cells (WBC), all those vital life indexes will return to normal; Certain patients without any infective factors other than HIV infection present high fever a few days after surgery, and then the body temperature gradually return to normal. However, general antibiotic treatment is hardly effective 
Table 1. Columns chi-square and Pearson correlation analysis for the corelation between the level of preoperative CD $4^{+} \mathrm{T}$ lymphocytes and perioperative sepsis occurrence.

\begin{tabular}{ccccccc}
\hline Preoperative $\mathrm{CD} 4{ }^{+}$T grouping & sepsis & nonsepsis & percent $\%$ & $\chi^{2}$ & $p$ & $r$ \\
\hline $\mathrm{A}(0-)$ & 19 & 4 & 82.609 & 23.680 & $<0.001$ & 0.987 \\
$\mathrm{~B}(100-)$ & 37 & 16 & 69.811 & & & \\
$\mathrm{C}(200-)$ & 17 & 25 & 40.476 & & & \\
$\mathrm{D}(350-)$ & 7 & 19 & 26.923 & & & \\
Total & 80 & 64 & 55.556 & & & \\
\hline
\end{tabular}

Table 2. Variance analysis for the single factor of sepsis during perioperative period.

\begin{tabular}{|c|c|c|c|c|c|}
\hline character & preoperative sepsis (A) & postoperative sepsis (B) & nonsepsis $(\mathrm{C})$ & $F$ & $p$ \\
\hline age & $37.57 \pm 11.37$ & $43.21 \pm 11.18$ & $43.11 \pm 13.90$ & 1.269 & 0.284 \\
\hline $\begin{array}{l}\text { Preoperative } \\
\text { CD4 count }\end{array}$ & $151.29 \pm 110.64$ & $161.14 \pm 128.45^{\#}$ & $276.97 \pm 137.91^{* \triangle}$ & 13.905 & $<0.001$ \\
\hline CD8 count & $619.93 \pm 392.54$ & $646.28 \pm 384.45$ & $810.62 \pm 447.37^{* \triangle}$ & 2.757 & 0.067 \\
\hline $\mathrm{CD} 4 / \mathrm{CD} 8$ & $0.27 \pm 0.26$ & $0.30 \pm 0.26$ & $0.45 \pm 0.36^{* \Delta}$ & 4.597 & 0.012 \\
\hline WBC & $7.77 \pm 3.57$ & $5.34 \pm 3.16^{\#}$ & $5.75 \pm 2.07^{*}$ & 4.456 & 0.013 \\
\hline HGB & $103.82 \pm 30.15$ & $114.46 \pm 24.03$ & $128.46 \pm 19.97^{* \Delta}$ & 9.667 & $<0.001$ \\
\hline PLT & $250.43 \pm 131.82$ & $211.27 \pm 105.71$ & $195.75 \pm 78.58$ & 1.870 & 0.158 \\
\hline ALB & $36.68 \pm 11.79$ & $42.54 \pm 14.19$ & $43.78 \pm 9.30$ & 1.328 & 0.269 \\
\hline $\begin{array}{l}\text { Postoperative } \\
\text { CD } 4^{+} \mathrm{T}\end{array}$ & $191.29 \pm 88.28$ & $152.05 \pm 136.13$ & $295.94 \pm 151.94^{\triangle}$ & 6.965 & 0.002 \\
\hline $\mathrm{CD}^{+} \mathrm{T}$ & $751.43 \pm 458.09$ & $554.21 \pm 304.53$ & $866.72 \pm 555.64^{\triangle}$ & 4.028 & 0.023 \\
\hline $\mathrm{CD} 4 / \mathrm{CD} 8$ & $0.32 \pm 0.31$ & $0.32 \pm 0.31$ & $0.42 \pm 0.27$ & 0.835 & 0.438 \\
\hline WBC & $7.09 \pm 5.62$ & $5.73 \pm 3.56$ & $6.30 \pm 1.83$ & 0.834 & 0.437 \\
\hline HGB & $99.02 \pm 21.77$ & $112.92 \pm 20.93^{\#}$ & $122.78 \pm 18.07^{* \Delta}$ & 6.222 & 0.003 \\
\hline PLT & $230.74 \pm 119.43$ & $181.05 \pm 102.30$ & $234.52 \pm 102.56^{\triangle}$ & 3.051 & 0.052 \\
\hline ALB & $36.28 \pm 13.26$ & $40.20 \pm 12.18$ & $40.30 \pm 6.13$ & 0.650 & 0.524 \\
\hline
\end{tabular}

Note: " significant between group C and group A; $\Delta$ significant between group B and group C; " significant between Group A and Group B; $\alpha=0.05$.

for some patients with obvious opportunistic infections, such as tuberculosis, pneumocystic Carinii pneumonia, fungal infections etc, which makes them prone to suffering septic shock and multiple organ failure. We suspected the diverse septic clinical manifestation of patients with HIV infections after surgery may be due to the different degrees of immune system impairment, the etiology of combined opportunistic infection and the stimulating reaction of organism to surgical trauma. HIV selectively invades cells with $\mathrm{CD} 4$ receptor, so $\mathrm{CD} 4^{+} \mathrm{T}$ lymphocytes are main target cells. Once infected by HIV, the functions of $\mathrm{CD}^{+} \mathrm{T}$ lymphocytes are impaired or even destroyed largely, which is the leading cause of immunodeficiency $[6,7]$. When the number of $\mathrm{CD}^{+}{ }^{+} \mathrm{T}$ lymphocytes are less than 200 cells $/ \mu 1$, patients may develop AIDS. At this period, the immune function of the patients is significantly compromised and consequently it makes patients prone to multiple opportunistic infections, along with a remarkably increased probability of postoperative infectious complications. The immune function is almost re- garded as normal among those patients with the number of $\mathrm{CD}^{+} \mathrm{T}$ lymphocytes no less than 350 cells $/ \mu 1$. Therefore, the number of $\mathrm{CD}^{+} \mathrm{T}$ lymphocytes less than 350 cells $/ \mu \mathrm{l}$ are treated as an indicator for clinical antiviral therapy. Assuming there is different susceptibility and severity to the sepsis, patients were divided into 4 groups based on their level of $\mathrm{CD}^{+} \mathrm{T}$ lymphocytes. Through Analysis of linear correlation between the level of CD $4^{+} \mathrm{T}$ lymphocytes and sepsis from each group, we find that the level of $\mathrm{CD}^{+} \mathrm{T}$ lymphocytes is negatively correlated with sepsis, which means the lower $\mathrm{CD}^{+} \mathrm{T}$ lymphocytes' level, the higher septic rate. Taking occurrence of sepsis from group of preoperative or postoperative sepsis and no perioperative sepsis as a single factor to test its variance analysis, we found that the level of $\mathrm{CD} 4{ }^{+} \mathrm{T}$ lymphocytes and hemachrome, the ratio of CD4 ${ }^{+} \mathrm{T} / \mathrm{CD} 8^{+} \mathrm{T}$ lymphocytes from group of no perioperative sepsis before surgery were all higher than the other two groups. The level of $\mathrm{CD}^{+} \mathrm{T}$ lymphocytes and hemachrome from group of no perioperative sepsis after surgery 
was higher than the other two groups.

\subsection{Operative Risk Evaluation}

The majority of our surgical patients were around 40 years old, most of them were male. About 1/4 patients had not been aware of their infection by HIV until diagnosed through routine examination before surgery. With the depletion of $\mathrm{CD}^{+} \mathrm{T}$ lymphocytes, some patients developed opportunistic infections, accompanied with symptoms of fever, emaciation, anemia etc. For instance, The low level of $\mathrm{CD}^{+} \mathrm{T}$ lymphocytes and hemachrome, the low ratio of CD4/CD8 $\mathrm{T}$ lymphocyte before surgery, the low level of platelet and hemachrome, the low ratio of CD4/CD8 $T$ lymphocyte, are all indexes that represent postoperative septic risk factors [8-10]. To evaluate surgical risk, those comprehensive factors, such as patients' general condition, the complexity of surgery, should be take into account. The treatments and surgical indication for those HIV infected patient with $\mathrm{CD} 4{ }^{+} \mathrm{T}$ lymphocytes more than $350 \mathrm{cell} / \mathrm{ul}$ are similar to those in general population. If the $\mathrm{CD} 4^{+} \mathrm{T}$ lymphocytes count ranges from 200 cells $/ \mu 1$ to 350 cells/ $\mu \mathrm{l}$, we should carefully determine whether patients have other concomitant diseases. If there are no other concomitant diseases, the extent of surgery should be appropriately reduced, and the vital signs should be remained stable during surgery so as to minimize the surgical damage to patients. However, if other concomitant diseases are found, preoperative risk evaluation is necessary, the surgery program should be performed on the basis of control of concomitant diseases. When the $\mathrm{CD}^{+} \mathrm{T}$ lymphocytes count is lower than 200 cells $/ \mu 1$, the surgery for HIV infective patients should be cautious; if surgery is the only strategy to save life or alleviate pain, patients and their relatives should be fully informed of the prognosis and peril of disease before the surgery. It should be noted that one patient with $\mathrm{CD}^{+} \mathrm{T}$ lymphocytes count only 6 cells/ $\mu$ l underwent emergency surgery due to intestinal perforation and peritonitis, the perforated and necrotic intestine was removed and proximal enterostomy was carried out. Even though postoperative wound infection was observed, that patient still discharged with favorable wound healing. In addition, we performed sigmoid colostomy, antifugal and antiretroviral treatment combined with sterile dressing change on one patient with $\mathrm{CD} 4^{+} \mathrm{T}$ lymphocytes as few as 34 cells/ $\mu \mathrm{l}$, perianal ulcer and cryptococcal meningitis. When patient's perianal ulcer recovered, sigmoid colostomy closure was carried out. At last, that patient was discharged with favorable outcome.

\subsection{Perioperative Treatment}

As tuberculosis, fungal infection and other opportunistic infections are commonly found among AIDS patients, the preoperative general condition should be rectified as early as possible. Those items include appropriate blood transfusion, fluid infusion, application of antibiotics or antifungal drugs for infection control. When $\mathrm{CD}^{+} \mathrm{T}$ lymphocytes count less than 200 cell/ul, sulfonamides and antifungal drugs ought to be applied for preventing from Pneumocystis pneumonia and other fungal infection. As bowel function paralysis after abdominal surgery may continue for 3 to 5 days, vein injection should be first taken as route of administration for tuberculosis and then return to oral till the recovery of bowel function. After terminating administration of antiretroviral drug the day of surgery, the normal administration should be resumed as soon as possible after surgery [11-13]. A gastric tube were retained at the time of abdominal surgery and closed temporarily after taking medicine. If patients have abdominal distention, some gastric juice can be drawn out one hour later after oral administration. Because resisting tuberculosis drug were used all the time, postoperative tuberculosis spread didn't occur.

\subsection{Prognosis of Sepsis in HIV Infected Patients}

With only wound infection and light systemic inflammatory response symptoms, HIV infective patients usually get better outcome under timely treatment. For those patients with tuberculosis or fungal infections, unless under emergency condition, surgery should not be started until these opportunistic infection are completely controlled; When emergency surgery are necessary in order to address the problem which non-operative treatment can't solve, adequate symptomatic should be given before surgery. After that, antibiotics and antifungal drugs through veinous access according to the length of operation is timely applied. In our study, among 80 perioperative septic cases, 5 patients had died from septic shock and MODS. In those 5 patients, 2 cases have serious pulmonary infection, and 3 cases have severe celiac infection. Other remaining 75 septic patients had recovered for prompt treatment. Therefore, surgery can address the key issues which threaten life or influence subsequent treatment on the basis of fully physical examination, operative risk evaluation and proper measures to control opportunistic infections. The quality of life and survival rate are greatly improved after surgery. In addition, the application of efficient antiretroviral drugs can keep HIV infected patients on the stable stage of disease and contribute to immune reconstruction which prolongs the survival.

\section{REFERENCES}

[1] Moore, C.C., Jacob, S.T., Pinkerton, R., et al. (2008) Point- 
of-care lactate testing predicts mortality of severe sepsis in a predominantly HIV type 1 - Infected patient population in Uganda. Clinical Infectious Diseases, 46, 215-222. doi:10.1086/524665

[2] Zaidi, A.K., Ganatra, H.A., Syed, S., et al. (2011) Effect of case management on neonatal mortality due to sepsis and pneumonia. BMC Public Health, 11, 13.

doi:10.1186/1471-2458-11-S3-S13

[3] Levinson, A.T., Casserly, B.P. and Levy, M.M. (2011) Reducing mortality in severe sepsis and septic shock. Seminars in Respiratory and Critical Care Medicine, 32, 195205. doi:10.1055/s-0031-1275532

[4] Lagu, T., Rothberg, M.B., Nathanson, B.H., et al. (2011) The relationship between hospital spending and mortality in patients with sepsis. Archives of Internal Medicine, 171, 292-299. doi:10.1001/archinternmed.2011.12

[5] Levy, M.M., Fink, M.P., Marshall, J.C., et al. (2003) 2001 $\mathrm{SCCM} / \mathrm{ESICM} / \mathrm{ACCP} / \mathrm{ATS} / \mathrm{SIS}$ international sepsis definitions conference. Critical Care Medicine, 31, 1250-1256. doi:10.1097/01.CCM.0000050454.01978.3B

[6] Jeremiah, L. Deneve, D.O., Jessica, G., et al. (2010) CD4 count is predictive of outcome in HIV-positive patients undergoing abdominal operations. American Journal of Surgery, 200, 694-700. doi:10.1016/j.amjsurg.2010.07.030

[7] Pereyra, F., Palmer, S., Miura, T., et al. (2009) Persistent low-level viremia in HIV-1 elite controllers and relationship to immunologic parameters. Journal of Infectious Diseases, 200, 984-990. doi:10.1086/605446
[8] Jain, S.B., Wig, N., Nagpal, S.J., et al. (2011) Evaluation of the current management protocols for prophylaxis against Pneumocystis jiroveci pneumonia and other opportunistic infections in patients living with HIV/AIDS. AIDS Care, 23, 1-5. doi:10.1080/09540121.2010.542129

[9] Sax, P.E., Sloan, C.E., Schackman, B.R., et al. (2010) Early antiretroviral therapy for patients with acute aids-related opportunistic infections: A cost-effectiveness analysis of ACTG A5164. HIV Clinical Trials, 11, 248-259. doi:10.1310/hct1105-248

[10] Kulkarni, S.V., Kairon, R., Sane, S.S., et al. (2009) Opportunistic parasitic infections in HIV/AIDS patients presenting with diarrhoea by the level of immunesuppression. Indian Journal of Medical Research, 130, 63-66.

[11] Hocqueloux, L., Prazuck, T., Avettand-Fenoel, V., et al. (2010) Long-term immunovirologic control following antiretroviral therapy interruption in patients treated at the time of primary HIV-1 infection. AIDS, 24, 1598-1601. doi:10.1097/QAD.0b013e32833b61ba

[12] Lemma, M., Vanelli, P., Beretta, L., et al. (1992) Cardiac surgery in HIV-positive intravenous drug addicts: Influence of cardiopulmonary bypass on the progression to AIDS. Journal of Thoracic and Cardiovascular Surgery, 40, 279282. doi:10.1055/s-2007-1022720

[13] Fasan, M.L., Morandi, E., Fociani, P., et al. (2003) AIDSassociated gastrointestinal lymphoma: Is there a role for surgery in the standard of care? Journal of Acquired Immune Deficiency Syndromes, 34, 345-347. doi:10.1097/00126334-200311010-00015 